\title{
émulations
}

\section{Julien Charles - La participation en actes. Entreprise, ville, association}

\section{Maité Juan}

Émulations - Revue de sciences sociales

2016, «Comptes rendus critiques, En ligne »

\section{Article disponible à l'adresse suivante}

https://ojs.uclouvain.be/index.php/emulations/article/view/7203

\section{Pour citer cet article}

Maité Juan, «Julien Charles — La participation en actes. Entreprise, ville, association », Émulations, en ligne. Mise en ligne le 25 juillet 2016.

DOI : 10.14428/emulations.cr.022

Distribution électronique : Université catholique de Louvain (Belgique) : ojs.uclouvain.be

(C) Cet article est mis à disposition selon les termes de la Licence Creative Commons Attribution, Pas d'Utilisation Commerciale 4.0 International. http://creativecommons.org/licenses/by-nc/4.0/

Éditeur : Émulations - Revue de sciences sociales / Presses universitaires de Louvain https://ojs.uclouvain.be/index.php/emulations

ISSN électronique : 1784-5734

UCL PRESSES

UNIVERSITAIRES 


\title{
Julien Charles - La participation en actes. Entreprise, ville, association
}

\begin{abstract}
Maité Juan ${ }^{1}$
Recensé : Julien Charles, La participation en actes. Entreprise, ville, association, Paris, Desclée de Brouwer, coll. «Solidarité et société », 2016, 168 p.

Voici un ouvrage dont l'originalité et le tour de force résident dans le défi de prendre le contrepied des études sociologiques s'attachant à étudier les vertus démocratiques de la participation, en se focalisant sur les charges pesant sur les individus dans leur engagement participatif. L'auteur fait le pari, largement tenu, d'éclairer la dimension contraignante et éprouvante de la participation, à travers une approche ethnographique fondée sur l'observation participante et la réalisation d'entretiens semi-directifs. On ne peut que saluer la parution de cet ouvrage, tiré d'une thèse de doctorat soutenue en 2012, fruit d'une cotutelle entre l'Université catholique de Louvain et l'École des hautes études en sciences sociales. La démarche pragmatique de la recherche s'inscrit dans la lignée conceptuelle de la sociologie des engagements de Thévenot (2006), qui vise à aborder les pouvoirs comme « des capacités à coordonner et à se coordonner » en liaison avec le déploiement des « mesures d'évaluation dont les instruments et méthodes sont explicitement destinés à des coordinations» (Thévenot, 2010). La démonstration de Julien Charles tire également parti de la distinction de Joëlle Zask (2011) entre "prendre part », " apporter une part » et « recevoir une part » et renverse la perspective en s'attachant à la part que les acteurs sont tenus de laisser de côté dans les dispositifs participatifs. Le fil rouge de l'ouvrage, rigoureusement suivi, est clair : «la participation impose des coûts » (p. 24). L'auteur se fonde sur un éventail contrasté de projets et dispositifs se revendiquant comme participatifs afin d'observer la manière dont ils induisent des exigences de mise en conformité aux cadres de la participation.

Le premier chapitre, qui apparaît comme le plus approfondi, étayé et convaincant, est consacré à « la mise en forme des contributions ». L'auteur s'attache à éclairer les formes de standardisation et de formatage de la participation au sein de deux milieux distincts, une usine belge ayant mis en place un dispositif de management participatif, le Caterpillar Production System (CPS), et un Plan communal de mobilité. À partir de l'étude pointue de deux dispositifs du CPS, les Continuous improvement Cards et les "dialogues de performance", l'auteur décortique de quelle manière la dynamique d'amélioration de la productivité, à partir de la conformation à des standards et à des
\end{abstract}

${ }^{1}$ Doctorante en sociologie au Cnam (LISE - Laboratoire interdisciplinaire de sociologie économique). 
indicateurs de performance, limite les contributions des acteurs. L'ajustement de l'acte de travail à des catégories et procédures apparaît comme une forme de déqualification, évacuant les connaissances pratiques et quotidiennes propres au savoir-faire des travailleurs. Ce cadrage de la participation constitue non seulement une entrave au déploiement de la critique, induisant un processus de dépolitisation, mais aussi une forme de violence. Le cas du Plan communal de mobilité dans la commune de Deuseau (nom fictif de commune), en Belgique, est tout aussi éclairant. Le comité de pilotage, impliquant les habitants aux côtés de membres de l'administration communale et d'un animateur de la participation, n'a pour unique visée que d'améliorer les supports cartographiques comme format légitime du Plan communal. La participation s'apparente ainsi à une collecte de renseignements qui ne prend pas en compte l'attachement des habitants à des mondes vécus et à des lieux communs.

Le deuxième chapitre, intitulé « La personne à l'épreuve de la participation » pénètre dans l'intériorité des individus afin d'interroger la charge exclusive imposée par ces mises en forme de la participation. S'inscrivant dans le sillage théorique de Zask, Sen, Zimmermann ou Eliasoph, l'auteur développe sa réflexion autour de la notion de capacité, définie à la fois comme " habitus » et comme apprentissage rendu possible par l'environnement. Julien Charles souligne, à juste titre, qu'au lieu d'atténuer l'inégale distribution des ressources entre les individus, en mettant en œuvre un accompagnement des participants dans l'acquisition des capacités requises pour la participation, ces dispositifs participatifs apparaissent peu pédagogiques et ne visent nullement à stimuler l'apprentissage collectif. L'introduction d'un cas de participation politisée offre un contraste édifiant qui laisse cependant apparaitre certaines dérives liées au modèle autogestionnaire. Julien Charles prend l'exemple d'une Maison médicale autogérée dont la genèse militante, sous la forme d'une boutique populaire, se fondait sur le «postulat d'égale compétence de tous à prendre part au projet dans toutes ses facettes » (p. 82). Sur la base d'un témoignage, l'auteur éclaire certaines contraintes inhérentes au modèle autogestionnaire telles que l'exigence d'expression critique, la distribution de l'autorité dans la communauté sans formalisation ni hiérarchie présentant un risque de dérive totalitaire, ou encore l'opacité des rapports sociaux au-delà du cercle de confiance du groupe fondateur. Pointant très justement que les dispositifs participatifs peuvent déboucher sur une "restriction de la personne à l'état de sujet politique » (p. 101), l'auteur montre comment le modèle autogestionnaire met en tension l'exigence de capacité délibérative d'un prétendu sujet politique et des modalités d'engagement plus affectives, intimes et quotidiennes. Le cas de la Maison médicale laisse apparaitre des modes divers de prise en compte des contributions personnelles et informelles des travailleurs à la gestion globale du projet, à la différence du Plan Communal de mobilité et du Caterpillar Production System.

Le troisième chapitre, intitulé " Une participation qui fait des choses », est consacré exclusivement au cas de la Bicycle Kitchen, un atelier de réparation de vélos, où des volontaires se mobilisent pour apprendre aux clients à réparer leur vélo. L'auteur 
montre, très pertinemment, que le projet d'empowerment se réduit à l'accomplissement d'une tâche. Il note que le point positif par rapport aux autres dispositifs participatifs étudiés réside dans le fait que ce projet laisse place à des « expérimentations tâtonnantes» (p.116) n'ayant pas à être conformes à des guides ou manuels. Cette improvisation et cette exploration des pistes induit une forme de participation plus libre et ludique. Cependant, cette centralité de l'usage, du «faire » détaché de tout partage de convictions ou de principes d'appartenance à un projet commun, conduit à une participation dépolitisée. Cette division entre les idéaux écologistes des volontaires et la pratique des clients centrée sur la remise en état des vélos dans une optique utilitaire fait perdre à la participation sa visée démocratique.

Le quatrième chapitre, intitulé « Des critiques de la participation : proposition d'amélioration des dispositifs participatifs » réaffirme que «les dispositifs de participation négligent les coûts de transformation qu'ils requièrent » (p.134) et met l'accent sur la reproduction de cette négligence par les analyses de la participation uniquement centrées sur les savoirs citoyens. Si l'auteur pointe la « clôture capacitaire » des projets participatifs, c'est pour mieux affirmer l'importance d'une « pédagogie de la démocratie et de l'émancipation » (p. 135).

En guise de conclusion, Julien Charles note judicieusement que les projets et dispositifs participatifs resteront des vecteurs d'exclusion et échoueront dans leur visée démocratique s'ils négligent ce qui est requis par la personne pour qu'elle contribue. De fait, c'est à condition que la participation reconnaisse l'inégalité des capacités entre les personnes et admette la pluralité des ressorts de l'engagement que pourrait se développer une "pédagogie de la participation » (p. 142). L'auteur achève sa réflexion en mettant en exergue quelques pistes fructueuses afin que la participation constitue un levier de critique et de politisation : la mise en œuvre de formats de communications adaptés aux modalités habituelles d'expression des participants, ainsi que l'expérimentation de dispositifs de participation faisant la jonction entre les pratiques et préoccupations des participants et des types plus conventionnels de participation.

Malgré la densité et la richesse de l'ouvrage, le manque de témoignages personnels étayant les réflexions stimulantes de l'auteur débouche parfois sur une certaine inconsistance de la démonstration, lorsqu'elle est moins ancrée dans l'étude fine du terrain. Bien que l'auteur souligne la nécessité d'analyser la participation à travers les motifs de l'engagement personnel, et non seulement sous l'angle des capacités requises, il n'approfondit pas cette piste de recherche et laisse le lecteur quelque peu sur sa faim. Enfin, l'approche critique aurait sans doute gagné à intégrer une discussion plus approfondie des travaux portant sur la démocratie participative ou le champ associatif afin d'étoffer le propos. L'auteur aurait ainsi pu mobiliser les analyses de Marrion Carrel (2013) autour des « artisans de la participation » dans les projets de rénovation urbaine, désignant des professionnels-militants qui animent les dispositifs participatifs afin de favoriser une coproduction de l'expertise avec les habitants. Carrel souligne également 
les dérives des dispositifs participatifs comme stratégie institutionnelle visant à pacifier la conflictualité sociale et non à émanciper, et invite à concevoir la citoyenneté comme « un apprentissage qui se joue dans l'intervalle entre individus et institutions » (p. 21). La mobilisation des analyses de Carrel aurait permis de nouer un dialogue fructueux autour non seulement des facteurs court-circuitant le débat public mais aussi des éléments générateurs d'une relation plus égalitaire, comme par exemple, les groupes de qualification mutuelle qui proscrivent le langage technique et complexe et valorisent les récits individuels permettant de placer le savoir technique au service des expériences individuelles. De la même manière, la démonstration de Julien Charles aurait pu se référer aux analyses de Julien Talpin sur les modes d'acquisition de la compétence civique, définie comme «l'ensemble des ressources cognitives, techniques, politiques, émotionnelles et pratiques dont disposent les citoyens pour intervenir dans l'espace public » (Talpin, 2010 : 96). L'auteur invite à évaluer le façonnement de la citoyenneté par le cadre politique et culturel dans lequel elle s'inscrit et à percevoir l'engagement délibératif comme un apprentissage collectif impliquant la maitrise d'un certain nombre de règles et pratiques. Les analyses tant de Carrel que de Talpin offrent à la fois un écho et un contrepoint qui auraient pu enrichir l'approche critique de l'ouvrage.

Enfin, un approfondissement de la question spécifique de l'engagement associatif pourrait enrichir la démonstration, notamment à travers l'analyse des modes de coexistence de la participation des bénévoles, des professionnels et des usagers. Laville et Sainsaulieu (2013) ont bien montré comment les processus de rationalisation professionnelle et gestionnaire au sein des associations comportent un risque d'essoufflement de l'engagement collectif, en bureaucratisant l'action associative et en rigidifiant les modes de participation des différentes parties prenantes. Ils ont notamment pointé l'importance de dispositifs délibératifs internes associant les différentes parties prenantes afin de réinjecter du participatif dans l'organisationnel. De la même manière, Laville et Eme ont développé le concept d'« espace public de proximité » pour désigner des initiatives solidaires se construisant comme des lieux de « co-construction de l'offre et de la demande » (Eme, Laville, 2004). L'ajustement de l'offre et de la demande entre prestataires, usagers et bénévoles s'opère par des dynamiques de débat, de délibérations et de décision collective qui sont des vecteurs de démocratisation.

Malgré ces quelques limites, qui sont en réalité des suggestions d'ouverture, l'ouvrage de Julien Charles représente une contribution précieuse, pertinente et aboutie pour l'ensemble des investigations prenant pour objet les processus participatifs. Il éclaire avec justesse de quelle manière la portée émancipatrice des dispositifs participatifs est pervertie par des cadrages et formatages qui contraignent les contributions des participants. La participation devient alors davantage un instrument de gestion et de pacification de la critique sociale qu'un vecteur de politisation et d'émancipation. L'ouvrage de Julien Charles apparait ainsi comme une invitation stimulante à débusquer 
les expérimentations innovantes s’attachant à mettre en œuvre une « pédagogie de la participation ».

\section{Bibliographie}

BolTANSKi L. (1990), L’amour et la justice comme compétences. Trois essais de sociologie de l’action, Paris, Métailié.

BoltANSKI L. (2009), De la critique, Paris, Gallimard.

BolTANSKI L. (2012), Énigmes et complots. Une enquête à propos d'enquêtes, Paris, Gallimard.

BoltANSKI L., Chiapello È. (1999), Le nouvel esprit du capitalisme, Paris, Gallimard.

BoltANSKI L., Esquerre A. (2014), Vers l'extrême. Extension des domaines de la droite, Paris, Dehors. 